\title{
On the appearance of Eisenstein series through degeneration
}

\author{
Daniel Garbin, Jay Jorgenson and Michael Munn
}

\begin{abstract}
Let $\Gamma$ be a Fuchsian group of the first kind acting on the hyperbolic upper half plane $\mathbb{H}$, and let $M=\Gamma \backslash \mathbb{H}$ be the associated finite volume hyperbolic Riemann surface. If $\gamma$ is parabolic, there is an associated (parabolic) Eisenstein series, which, by now, is a classical part of mathematical literature. If $\gamma$ is hyperbolic, then, following ideas due to Kudla-Millson, there is a corresponding hyperbolic Eisenstein series. In this article, we study the limiting behavior of parabolic and hyperbolic Eisenstein series on a degenerating family of finite volume hyperbolic Riemann surfaces. In particular, we prove the following result. If $\gamma \in \Gamma$ corresponds to a degenerating hyperbolic element, then a multiple of the associated hyperbolic Eisenstein series converges to parabolic Eisenstein series on the limit surface.
\end{abstract}

Mathematics Subject Classification (2000). 11M36, 30F35, 11F72.

Keywords. Hyperbolic Eisenstein series, degenerating Riemann surfaces, and counting functions.

\section{Introduction}

1.1. Spectral expansions. Let $M=\Gamma \backslash \mathbb{H}$ be a finite volume hyperbolic Riemann surface, realized as the quotient of the hyperbolic upper half plane $\mathbb{H}$ by a discrete subgroup $\Gamma$ of $\mathrm{PSL}_{2}(\mathbb{R})$. Let $\Delta_{M}$ denote the Laplacian, associated to the hyperbolic metric, which acts on the space of smooth functions on $M$. For the sake of our discussion, consider the corresponding heat kernel $K_{M}(t ; z, w)$, which is a function of $t \in \mathbb{R}^{+}$and $z, w \in M$. If $M$ is compact, then the heat kernel admits the spectral expansion

$$
K_{M}(t ; z, w)=\sum_{n=0}^{\infty} e^{-\lambda_{M, n} t} \phi_{M, n}(z) \overline{\phi_{M, n}(w)}
$$

where $\left\{\phi_{M, n}\right\}$ is a complete orthonormal basis of eigenfunctions of the $\Delta_{M}$ with corresponding (non-negative) eigenvalues $\lambda_{M, n}$. If $M$ is non-compact, then the spectral expansion of the heat kernel takes a very different form. More specifically, let $\{P\}$ denote the finite set of $\Gamma$-inequivalent cusps, and $E_{\mathrm{par} ; M, P}(s, z)$ be the (parabolic) Eisenstein series on $M$ corresponding to $P$. Then, in this case, the spectral expansion 
of the heat kernel on $M$ is the identity

$$
\begin{aligned}
& K_{M}(t ; z, w) \\
& =\sum_{n=0}^{\infty} e^{-\lambda_{M, n} t} \phi_{M, n}(z) \overline{\phi_{M, n}(w)} \\
& \quad+\frac{1}{4 \pi} \sum_{P} \int_{-\infty}^{\infty} e^{-\left(r^{2}+1 / 4\right) t} E_{\mathrm{par} ; M, P}(1 / 2+i r, z) \overline{E_{\mathrm{par} ; M, P}(1 / 2+i r, w)} d r .
\end{aligned}
$$

Recall now that any finite volume hyperbolic Riemann surface $M_{0}$ can be realized as one component of a degenerating sequence of compact hyperbolic Riemann surfaces $M_{\ell}$. In this setting, it has been shown that the hyperbolic heat kernels on $M_{\ell}$ converge to the hyperbolic heat kernel on $M_{0}$; see [13] and [15]. With the heat kernel convergence result in mind, one immediately has from (1) and (2) the following natural question: How does one see the emergence of the Eisenstein series in (2) through degeneration? More precisely, does there exist a naturally defined sequence of functions $h_{\ell}(s, z)$ on $M_{\ell}$ which converges to the Eisenstein series $E_{\mathrm{par} ; M_{0}, P}(s, z)$ on $M_{0}$ ?

1.2. Spectral theory on degenerating Riemann surfaces. The problem of studying the asymptotic behavior of spectral theory on degenerating Riemann surfaces of finite volume has received considerable attention in the literature. In [5], Hejhal developed the theory of degenerating $b$-groups and obtained, among other results, the lead asymptotics of spectral counting functions. An improvement of the error term in the case the degenerating surfaces are compact was proved in [8]. From [2], one has a construction of degenerating hyperbolic Riemann surfaces of finite volume by first constructing families of degenerating algebraic curves, from which one can utilize the uniformization theorem in order to obtain degenerating families of Riemann surfaces of finite volume. In [9], the approach from [2] is used to study spectral invariants associated to the canonical and Arakelov metrics. Beginning in [13], Huntley, Jorgenson and Lundelius used the methodology from [2] to study hyperbolic spectral theory through degeneration. These authors obtained numerous result, including: Convergence of heat kernels [13]; asymptotic behavior of heat traces and Selberg zeta functions [16]; convergence of relative spectral functions [14]; asymptotic behavior of counting functions [15]; asymptotic behavior of weighted counting functions (Riesz sums) [6]. In all these articles, the results apply to non-compact degenerating families as well as compact families. Further results concerning eigenvalue and eigenfunction convergence have been obtained by Judge in [17] and [18], and Wolpert used degenerating techniques to study the problem of existence of $L^{2}$ eigenfunctions on general finite volume hyperbolic Riemann surfaces. More recently, in [11], it was shown that 
one can use the results from [16] to study other metrics, namely it was shown that the metric on Teichmüller space induced from the canonical metric is not complete.

In brief, there is a vast literature addressing problems in the study of spectral theory on degenerating finite volume Riemann surfaces. Further problems exist, and as mathematical development demonstrates, new results are interesting for their own sake as well as for potential applications to other fields.

1.3. The main results. Throughout this article we refer to the Eisenstein series $E_{\text {par; } M, P}(s, z)$ in (2) as parabolic Eisenstein series since each such series is associated to a parabolic element of the uniformizing group $\Gamma$. In [20], the authors defined a hyperbolic Eisenstein series $E_{\mathrm{hyp} ; M, \gamma}(s, z)$ associated to any hyperbolic element $\gamma \in \Gamma$. We will summarize both definitions in Sections 2.3 and 2.4. In addition, as we will recall below, a degenerating family of hyperbolic Riemann surfaces $M_{\ell}$ has two types of hyperbolic elements: Non-degenerating elements, which are those that converge to hyperbolic elements in the Fuchsian group of the limit surface, and degenerating elements, which are those whose associated geodesics have lengths that converge to zero.

Precise definitions and references to all concepts will be given in Section 2 below. However, with these comments made, we are able to state the main result of the paper.

Main Theorem. Let $M_{\ell}$ be a degenerating family of hyperbolic Riemann surfaces of finite volume, with limit surface $M_{0}$.

i) Let $E_{\mathrm{hyp} ; M_{\ell}, \gamma}(s, z)$ be the hyperbolic Eisenstein series on $M_{\ell}$ associated to the hyperbolic element $\gamma$. If $\gamma$ corresponds to a non-degenerating hyperbolic element, then

$$
\lim _{\ell_{\gamma} \rightarrow 0} E_{\mathrm{hyp} ; M_{\ell}, \gamma}(s, z)=E_{\mathrm{hyp} ; M_{0}, \gamma}(s, z) .
$$

ii) Let $E_{\mathrm{par} ; M_{\ell}, P}(s, z)$ be the parabolic Eisenstein series on $M_{\ell}$ associated to the cusp $P$. Then

$$
\lim _{\ell_{\gamma} \rightarrow 0} E_{\mathrm{par} ; M_{\ell}, P}(s, z)=E_{\mathrm{par} ; M_{0}, P}(s, z)
$$

iii) Let $E_{\mathrm{hyp} ; M_{\ell}, \gamma}(s, z)$ be the hyperbolic Eisenstein series on $M_{\ell}$ associated to the hyperbolic element $\gamma$, whose geodesic has length $\ell_{\gamma}$. If $\gamma$ corresponds to a degenerating hyperbolic element which results in the new cusp $P$, then

$$
\lim _{\ell_{\gamma} \rightarrow 0} \ell_{\gamma}^{-s} E_{\mathrm{hyp} ; M_{\ell}, \gamma}(s, z)=E_{\mathrm{par} ; M_{0}, P}(s, z) .
$$

In all instances, the convergence is uniform on compact subsets of $M_{0}$ bounded away from the developing cusps, and in half-planes of the form $\operatorname{Re}(s) \geq 1+\delta$ for any $\delta>0$. 
Part (iii) answers the question posed above, namely to determine a naturally defined sequence of functions on a degenerating family $M_{\ell}$ of hyperbolic Riemann surfaces whose limit is the parabolic Eisenstein series associated to the newly developed cusps.

Explanation of (iii). In order to keep the statement of part (iii) manageable, we employed a slight abuse of notation, which we now explain. If $M_{\ell}$ has a single pinching geodesic which is separating, then the limit surface $M_{0}$ has two components, which we denote by $M_{0 ; 1}$ and $M_{0 ; 2}$, each with a single newly formed cusp, denoted by $P_{1}$ and $P_{2}$. In this case, the right-hand side of (iii) depends on the location of the point $z$ : If $z \in M_{0 ; 1}$ (resp. $z \in M_{0 ; 2}$ ), then the right-hand side of (iii) signifies the function $E_{\mathrm{par} ; M_{0 ; 1}, P_{1}}(s, z)$ (resp. $E_{\mathrm{par} ; M_{0: 2} ; P_{2}}(s, z)$ ). If $M_{\ell}$ has a single pinching geodesic which is non-separating, then the limit surface $M_{0}$ has one component with two newly formed cusps, denoted by $P_{1}$ and $P_{2}$. In this case, the right-hand side of (iii) signifies the function $E_{\mathrm{par} ; M_{0}, P_{1}}(s, z)+E_{\mathrm{par} ; M_{0}, P_{2}}(s, z)$. To consider the general case when $M_{\ell}$ has several pinching geodesics, then one simply iterates the results from the Main Theorem by pinching one geodesic at a time.

1.4. Outline of the paper. In Section 2, we establish notation and recall various known results. Perhaps the most important ingredients for our analysis are the definitions of parabolic and hyperbolic counting functions, and the realization that parabolic and hyperbolic Eisenstein series can be expressed as Stieltjes integrals of these counting functions. In Section 3, we study the asymptotic behavior of the counting functions from Section 2 through degeneration. With these results, we conclude by proving the Main Theorem in Section 4.

\section{Background material}

2.1. Basic notation. Let $M$ be a finite volume hyperbolic Riemann surface. By this we mean there exists a Fuchsian group of the first kind $\Gamma$ acting on the hyperbolic upper half plane $\mathbb{H}$ such that $M$ and $\Gamma \backslash \mathbb{H}$ are isometric. Hence, we write $M=\Gamma \backslash \mathbb{H}$. As is common, we realize $\mathbb{H}$ as $\{z \in \mathbb{C} \mid \operatorname{Im}(z)>0\}$. Writing $z=x+i y$, then the hyperbolic metric $\mu_{\text {hyp }}$ and hyperbolic Laplacian $\Delta_{\text {hyp }}$ can be expressed as

$$
\mu_{\text {hyp }}=\frac{d x^{2}+d y^{2}}{y^{2}} \text { and } \Delta_{\text {hyp }}=-y^{2}\left(\frac{\partial^{2}}{\partial x^{2}}+\frac{\partial^{2}}{\partial y^{2}}\right) .
$$

Under the change of coordinates $x=e^{\rho} \cos \theta$ and $y=e^{\rho} \sin \theta$, the hyperbolic metric and hyperbolic Laplacian are given by

$$
\mu_{\text {hyp }}=\frac{d \rho^{2}+d \theta^{2}}{\sin ^{2} \theta} \quad \text { and } \quad \Delta_{\text {hyp }}=-\sin ^{2} \theta\left(\frac{\partial^{2}}{\partial \rho^{2}}+\frac{\partial^{2}}{\partial \theta^{2}}\right) .
$$


In a slight abuse of notation, we will at times in this article identify $M$ with a fundamental domain (say, a Ford domain, bounded by geodesic paths) and identify points on $M$ with their pre-images in $\mathbb{H}$.

2.2. Counting functions. Let $\gamma \in \Gamma$ be a primite hyperbolic element. As usual, primitive means that if $\gamma_{0} \in \Gamma$ and $\gamma_{0}^{n}=\gamma$ for some integer $n$, then $n= \pm 1$. By hyperbolic, one means that $\gamma$ can be conjugated in $\mathrm{PSL}_{2}(\mathbb{R})$ to a non-identity diagonal matrix, which we write as

$$
\gamma=\left(\begin{array}{ll}
e^{\ell_{\gamma} / 2} & 0 \\
0 & e^{-\ell_{\gamma} / 2}
\end{array}\right)
$$

where $\ell_{\gamma}$ denotes the length of the closed geodesic on $M$ in the homotopy class determined by $\gamma$. Let $\Gamma_{\gamma}$ be the stabilizer in $\Gamma$ of $\gamma$, and we assume that $\Gamma_{\gamma}$ is generated by $\gamma$; it is easily shown that $\Gamma_{\gamma}$ is isomorphic to $\mathbb{Z}$. Choose a realization of $\Gamma$ in $\operatorname{PSL}_{2}(\mathbb{R})$ such that $\gamma$ is diagonal. Then the geodesic in $\mathbb{H}$ fixed by $\gamma$ is the line $\mathcal{L}_{0}=\{\operatorname{Re}(z)=0\} \cap \mathbb{H}$. For any point $z \in M$, which we lift to a point $z \in \mathbb{H}$, let $d_{\text {hyp }}\left(z, \mathscr{L}_{0}\right)$ denote the geodesic distance from $z$ to $\mathscr{L}_{0}$. With all this, we define the hyperbolic counting function as

$$
N_{\text {hyp } ; M, \gamma}(T ; z)=\operatorname{card}\left\{\eta \in \Gamma_{\gamma} \backslash \Gamma \mid d_{\text {hyp }}\left(\eta z, \mathcal{L}_{0}\right)<T\right\} .
$$

Equivalently, one can count the number of geodesic paths from $z \in M$ to the closed geodesic on $M$ in the homotopy class determined by $\gamma$; see Figure 1 . By following the

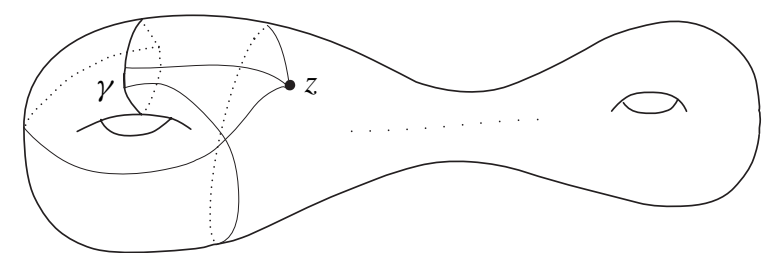

Figure 1. Geodesic paths from a point to a closed geodesic.

method of proof in Lemma 1.4 of [15] (see also [21]), which simply utilizes elementary hyperbolic geometric considerations, we can establish the following bound. For any point $z \in M$ with injectivity radius $r$, and any $u>T_{0}>r$, we have

$$
N_{\text {hyp } M, \gamma}(u ; z) \leq N_{\text {hyp } M, \gamma}\left(T_{0} ; z\right)+\frac{\sinh ^{2}\left(\frac{u+r}{2}\right)-\sinh ^{2}\left(\frac{T_{0}-r}{2}\right)}{\sinh ^{2}\left(\frac{r}{2}\right)} .
$$

For the sake of completeness and convenience of the reader, we now give a proof of (3). 
Let $B_{z}(T)$ denote the hyperbolic ball of hyperbolic radius $T$ centered at $z$. Let $\left\{\eta_{k}\right\} \subset \Gamma_{\gamma} \backslash \Gamma$ be a maximal collection of elements such that $\eta_{k} z \in B_{z}(u) \backslash B_{z}\left(T_{0}\right)$. Note that

$$
\bigcup_{k} B_{\eta_{k} z}(r) \subset B_{z}(u+r) \backslash B_{z}\left(T_{0}-r\right),
$$

so then

$$
\operatorname{vol}_{\text {hyp }}\left(\bigcup_{k} B_{\eta_{k} z}(r)\right) \leq \operatorname{vol}_{\text {hyp }}\left(B_{z}(u+r)\right)-\operatorname{vol}_{\text {hyp }}\left(B_{z}\left(T_{0}-r\right)\right),
$$

where vol $_{\text {hyp }}$ denotes the hyperbolic volume. Since $r$ is the injectivity radius at $z$, we then have

$$
\sum_{k} \operatorname{vol}_{\text {hyp }}\left(B_{\eta_{k} z}(r)\right) \leq \operatorname{vol}_{\text {hyp }}\left(B_{z}(u+r)\right)-\operatorname{vol}_{\text {hyp }}\left(B_{z}\left(T_{0}-r\right)\right) .
$$

By computing the volume of geodesic balls, in $\mathbb{H}$, we have that

$$
\begin{aligned}
& \operatorname{card}\left\{\eta \in \Gamma_{\gamma} \backslash \Gamma \mid \eta z \in B_{z}(u) \backslash B_{z}\left(T_{0}\right)\right\} \cdot 4 \pi \sinh ^{2}(r / 2) \\
& \leq 4 \pi\left(\sinh ^{2}\left(\frac{u+r}{2}\right)-\sinh ^{2}\left(\frac{T_{0}-r}{2}\right)\right) .
\end{aligned}
$$

Since

$$
\begin{aligned}
\operatorname{card}\left\{\eta \in \Gamma_{\gamma} \backslash \Gamma \mid \eta z \in B_{z}(u) \backslash B_{z}\left(T_{0}\right)\right\}= & \operatorname{card}\left\{\eta \in \Gamma_{\gamma} \backslash \Gamma \mid \eta z \in B_{z}(u)\right\} \\
& -\operatorname{card}\left\{\eta \in \Gamma_{\gamma} \backslash \Gamma \mid \eta z \in B_{z}\left(T_{0}\right)\right\},
\end{aligned}
$$

we get the desired result, namely the bound

$$
\begin{aligned}
& \operatorname{card}\left\{\eta \in \Gamma_{\gamma} \backslash \Gamma \mid \eta z \in B_{z}(u)\right\} \\
& \quad \leq \operatorname{card}\left\{\eta \in \Gamma_{\gamma} \backslash \Gamma \mid \eta z \in B_{z}\left(T_{0}\right)\right\}+\frac{\sinh ^{2}\left(\frac{u+r}{2}\right)-\sinh ^{2}\left(\frac{T_{0}-r}{2}\right)}{\sinh ^{2}\left(\frac{r}{2}\right)},
\end{aligned}
$$

thus completing the proof of (3).

Consider now a parabolic element $\gamma \in \Gamma$, which, by conjugation in $\mathrm{PSL}_{2}(\mathbb{R})$, we may assume

$$
\gamma=\left(\begin{array}{cc}
1 & \omega \\
0 & 1
\end{array}\right)
$$

where $\omega$ is referred to as the width of the cusp associated to $\gamma$. Let $\Gamma_{\infty}$ denote the stabilizer in $\Gamma$ of $\gamma$, and without loss of generality we may assume that $\gamma$ generates $\Gamma_{\infty}$. Choose and fix any point $z \in M$, which we lift to a point $z \in \mathbb{H}$. Elementary considerations show that one can choose $y_{0} \in \mathbb{R}$ sufficiently large so that $y_{0}>\operatorname{Im}(\eta z)$ for all $\eta \in \Gamma$. Let $\mathcal{L}_{y_{0}}$ be the horocyclic line in $\mathbb{H}$ defined by $\left\{\operatorname{Im}(z)=y_{0}\right\}$. For any 
point $z \in M$, which we lift to a point $z \in \mathbb{H}$, let $d_{\text {hyp }}\left(z, \mathcal{L}_{y_{0}}\right)$ denote the geodesic distance from $z$ to $\mathcal{L}_{y_{0}}$. With all this, we define the parabolic counting function associated to $\gamma$ and $y_{0}$ to be

$$
N_{\text {par } ; M, P}\left(T ; z, y_{0}\right)=\operatorname{card}\left\{\eta \in \Gamma_{\infty} \backslash \Gamma \mid d_{\text {hyp }}\left(\eta z, \mathscr{L}_{y_{0}}\right)<T\right\} .
$$

Observe that when defining the parabolic counting function, we needed to use the length from $z$ to a horocyclic line $\mathcal{L}_{y_{0}}$ since the cusp is at infinite distance. Such considerations are not necessary when defining the hyperbolic counting function. Finally, as with (3), the arguments from [21] apply to yield the following bound. For any point $z \in M$ with injectivity radius $r$, and any $u>T_{0}>r$, we have

$$
N_{\text {par } ; M, P}\left(u ; z, y_{0}\right) \leq N_{\text {par }, M, P}\left(T ; z, y_{0}\right)+\frac{\sinh ^{2}\left(\frac{u+r}{2}\right)-\sinh ^{2}\left(\frac{T_{0}-r}{2}\right)}{\sinh ^{2}\left(\frac{r}{2}\right)} .
$$

The proof of (4) is similar to the proof of (3) given above.

2.3. Parabolic Eisenstein series. By now, the study of parabolic Eisenstein series associated to a cusp $P$ on a non-compact, finite volume hyperbolic Riemann surface $M$ is a classical aspect of mathematics (see, for example, [4], [7] or [19]). To recall, for any $z \in \mathbb{H}$ and $s \in \mathbb{C}$ with $\operatorname{Re}(s)>1$, we define the parabolic Eisenstein series $E_{\text {par; } M, P}(s, z)$ by

$$
E_{\mathrm{par} ; M, P}(s, z)=\omega^{-s} \sum_{\eta \in \Gamma_{\infty} \backslash \Gamma}(\operatorname{Im} \eta z)^{s}
$$

It is standard in the mathematical literature to normalize cusps so that the width $\omega$ is equal to one. We will work slightly more generally and, as a result, include the multiplicative factor of $\omega^{-s}$ in (5). For any point $z \in \mathbb{H}$ and $y_{0} \in \mathbb{R}$ with $\operatorname{Im}(z)<y_{0}$, we have that

$$
d_{\text {hyp }}\left(z, \mathcal{L}_{y_{0}}\right)=\int_{\operatorname{Im}(z)}^{y_{0}} \frac{d y}{y}=\log \left(\frac{y_{0}}{\operatorname{Im}(z)}\right),
$$

so then

$$
(\operatorname{Im}(z))^{s}=y_{0}^{s} \exp \left(-s \cdot d_{\text {hyp }}\left(z, \mathcal{L}_{y_{0}}\right)\right) .
$$

With this observation, we can express the parabolic Eisenstein series (5) as a Stieltjes integral, namely

$$
E_{\mathrm{par} ; M, P}(s, z)=\left(y_{0} / \omega\right)^{s} \int_{0}^{\infty} e^{-s u} d N_{\mathrm{par} ; M, P}\left(u ; z, y_{0}\right) .
$$

Observe that the integral in (6) depends on the choice of $y_{0}$ through the parabolic counting function; however, after multiplying by $y_{0}^{s}$ the product itself is independent 
of $y_{0}$. As we will see, one can use (4) and (6) to prove the well-known result that (5) converges uniformly and absolutely for $\operatorname{Re}(s)>1$. Though not needed in this article, we state, for the sake of completeness, the classical differential equation satisfied by the parabolic Eisenstein series, which is the identity

$$
\Delta E_{\mathrm{par} ; M, P}(s, z)=s(1-s) E_{\mathrm{par}, M, P}(s, z) .
$$

2.4. Hyperbolic Eisenstein series. Let $M=\Gamma \backslash \mathbb{H}$ be any finite volume, compact or non-compact, hyperbolic Riemann surface, and let $\gamma$ be any hyperbolic element of $\Gamma$. As in Section 2.2, we assume that $\Gamma$ has been conjugated by an element in $\mathrm{PSL}_{2}(\mathbb{R})$ so that $\gamma$ is diagonal. We will use the change of coordinates $z=e^{\rho} e^{i \theta}$ and write $\theta(z)=\theta$. With this notation, we formally define the hyperbolic Eisenstein series $E_{\mathrm{hyp} ; M, \gamma}(s, z)$ by

$$
E_{\mathrm{hyp} ; M, \gamma}(s, z)=\sum_{\eta \in \Gamma_{\gamma} \backslash \Gamma}(\sin \theta(\eta z))^{s} .
$$

The hyperbolic metric in the $(\rho, \theta)$ coordinates was given in Section 2.1, from which one can easily show that

$$
d_{\text {hyp }}\left(z, \mathcal{L}_{0}\right)=|\log (\csc \theta(z)+\cot \theta(z))|,
$$

which can be used to obtain the relation

$$
\sin (\theta(z)) \cdot \cosh \left(d_{\text {hyp }}\left(z, \mathscr{L}_{0}\right)\right)=1,
$$

so then we can write (7) as

$$
E_{\mathrm{hyp} ; M, \gamma}(s, z)=\sum_{\eta \in \Gamma_{\gamma} \backslash \Gamma}\left(\cosh \left(d_{\mathrm{hyp}}\left(\eta z, \mathcal{L}_{0}\right)\right)\right)^{-s} .
$$

We can express the hyperbolic Eisenstein series (7) as a Stieltjes integral, namely

$$
E_{\mathrm{hyp} ; M, \gamma}(s, z)=\int_{0}^{\infty}(\cosh u)^{-s} d N_{\mathrm{hyp} ; M, \gamma}(u ; z) .
$$

A by-product of the computations from Section 4 is that by combining (3) and (8), we can show that the series defining the hyperbolic Eisenstein series (7) converges uniformly and absolutely for $\operatorname{Re}(s)>1$ (see also [20] and [22]). Also, using the computations from Section 2.1, one can easily verify the differential equation

$$
\Delta E_{\mathrm{hyp} ; M, \gamma}(s, z)=s(1-s) E_{\mathrm{hyp} ; M, \gamma}(s, z)+s^{2} E_{\mathrm{hyp} ; M, \gamma}(s+2, z),
$$

which is given in [20], [22] and [24] (Lemma 3.2). 
2.5. Degenerating families of Riemann surfaces. The discussion in this section is taken from [16] and is repeated here for the convenience of the reader.

In [13] and [14] the authors gave a construction of a degenerating family $M_{\ell}$ of either compact or non-compact hyperbolic Riemann surfaces of finite volume. The construction of the family $M_{\ell}$ allows one to define unambiguously various notions such as the tracking of points through degeneration and the idea of points not contained on the degenerating geodesics. The reader is referred to these articles for complete details, which will be assumed here.

Let $M_{\ell}$ be a degenerating family of connected, hyperbolic Riemann surfaces with $p$ degenerating geodesics, with $\ell$ denoting a $p$-tuple corresponding to the lengths of the degenerating geodesics. To say that $\ell$ approaches zero means that the length of each degenerating geodesic is approaching zero. Although each $M_{\ell}$ is connected when $\ell>0$, the limit surface $M_{0}$ need not be connected and, indeed, the number of cusps on $M_{0}$ is equal to the number of cusps on $M_{\ell}$ plus $2 p$.

For $\ell>0$, let $\mathcal{C}_{\ell}$ denote the hyperbolic infinite cylinder with simple, closed geodesic of length $\ell$. A convenient fundamental domain for $\mathcal{C}_{\ell}$ in $\mathbb{H}$ is

$$
\{r \exp (i \alpha) \mid 1 \leq r<\exp (\ell), 0<\alpha<\pi\},
$$

with hyperbolic metric induced from $\mathbb{H}$ and uniformizing group $\{\exp (k \ell) \mid k \in \mathbb{Z}\}$ which acts on $\mathbb{H}$ by multiplication. For any $\varepsilon>0$, let $\mathcal{C}_{\ell, \varepsilon}$ denote the symmetric submanifold of $\mathcal{C}_{\ell}$ about the geodesic defined by $\gamma$ with total volume equal to $\varepsilon$. A model for $\mathcal{C}_{\ell, \varepsilon}$ in (10) is obtained by adding the restriction

$$
\cot ^{-1}(\varepsilon /(2 \ell))<\alpha<\pi-\cot ^{-1}(\varepsilon /(2 \ell)) .
$$

An easy calculation shows that the length of each boundary component of $\mathcal{C}_{\ell, \varepsilon}$ is $\left(\ell^{2}+\varepsilon^{2} / 4\right)^{1 / 2}$. If $\varepsilon_{1}>\varepsilon_{0}$, then the distance from the boundary of $\mathcal{C}_{\ell, \varepsilon_{1}}$ and $\mathcal{C}_{\ell, \varepsilon_{0}}$ can be shown to be

$$
d_{\text {hyp }}\left(\partial \mathcal{C}_{\ell, \varepsilon_{0}}, \partial \mathcal{C}_{\ell, \varepsilon_{1}}\right)=\log \left(\left(\varepsilon_{1}+\sqrt{\left(\varepsilon_{1}^{2}+4 \ell^{2}\right)}\right) /\left(\varepsilon_{0}+\sqrt{\left(\varepsilon_{0}^{2}+4 \ell^{2}\right)}\right)\right) .
$$

From [23] we have that for any $0<\varepsilon<1 / 2$, the surface $\mathcal{C}_{\ell, \varepsilon}$ embeds isometrically into $M_{\ell}$. The surface $M_{0}$ contains $2 p$ embedded copies of $\mathcal{C}_{0, \varepsilon}$ which is the limit of $\mathcal{C}_{\ell, \varepsilon} \subset M_{\ell}$. One can model $\mathcal{C}_{0, \varepsilon}$ as two copies of a symmetric neighborhood of the origin in the punctured unit disc with its complete hyperbolic metric. From [1] we have that the family of hyperbolic metrics converges uniformly on $M_{\ell} \backslash \mathcal{C}_{\ell, \varepsilon}$.

2.6. A Stieltjes integral inequality. A key component in our analysis is an integral inequality for Stieltjes integrals, which we quote from [10] and, for the sake of completeness, we state here. Let $F$ be a real valued, smooth, decreasing function defined for $u>0$ and let $g_{1}, g_{2}$ be real valued, non decreasing functions defined for 
$u \geq a>0$ and satisfying $g_{1}(u) \leq g_{2}(u)$ for $u \geq a$. Then, the following inequality of Stieltjes integrals

$$
\int_{a}^{\infty} F(u) d g_{1}(u)+F(a) g_{1}(a) \leq \int_{a}^{\infty} F(u) d g_{2}(u)+F(a) g_{2}(a)
$$

holds, provided both integrals exist.

\section{Convergence of counting functions}

In this section we will establish the limiting behavior of the counting functions $N_{\text {par } ; M_{\ell}, P}$ and $N_{\text {hyp } ; M_{\ell}, \gamma}$ on a degenerating family of finite volume hyperbolic Riemann surfaces $M_{\ell}$. For simplicity, we will assume that $M_{\ell}$ has a single family of degenerating geodesics; the more general situation is easily obtained from the arguments presented here with only a slight modification of notation.

Throughout this article we make use of the following fundamental result which we cite without proof from [1], stated as Theorem 8, page 37.

3.1. Proposition. With notation as above, the hyperbolic metrics on the degenerating family $M_{\ell}$ converge to the hyperbolic metric on $M_{0}$. Furthermore, the convergence is uniform on compact subsets of $M_{0}$ bounded away from the developing cusps.

We refer the reader to [1] for a complete proof of Proposition 3.1. Building on this result, we consider the convergence of the hyperbolic and parabolic counting functions through degeneration.

3.2. Lemma. With notation as above, we have the following limits:

a) If $\gamma$ does not correspond to a degenerating hyperbolic element, then

$$
\lim _{\ell \rightarrow 0} N_{\text {hyp } ; M_{\ell}, \gamma}(T ; z)=N_{\text {hyp } ; M_{0}, \gamma}(T ; z) ;
$$

b) For any cusp $P$, we have

$$
\lim _{\ell \rightarrow 0} N_{\mathrm{par} ; M_{\ell}, P}\left(T ; z, y_{0}\right)=N_{\mathrm{par} ; M_{0}, P}\left(T ; z, y_{0}\right) .
$$

In all instances, the convergence is uniform on compact subsets of $M_{0}$ bounded away from the developing cusps. 
Proof. Choose $\varepsilon_{1}$ sufficiently small so that the point $z$ lies in $M_{\ell} \backslash \mathcal{C}_{\ell, \varepsilon_{1}}$. Now choose $\varepsilon_{0}<\varepsilon_{1}$ so that the distance from the boundary of $\mathcal{C}_{\ell, \varepsilon_{0}}$ to the boundary of $\mathcal{C}_{\ell, \varepsilon_{1}}$ is greater than $T$. Clearly, any geodesic path from $z$ to the non-pinching geodesic $\gamma$ with length bounded by $T$ necessarily lies entirely in the $M_{\ell} \backslash \mathcal{C}_{\ell, \varepsilon_{0}}$. From Proposition 3.1, we know that the family of hyperbolic metrics converge uniformly away on $M_{\ell} \backslash \mathcal{C}_{\ell, \varepsilon_{0}}$, which proves part (a).

The convergence statement asserted in (b) follows from a similar argument.

3.3. Lemma. With notation as above, let

$$
g\left(y_{0}, \ell\right)=\log \left(\frac{\omega}{y_{0} \ell}+\sqrt{\left(\frac{\omega}{y_{0} \ell}\right)^{2}+1}\right)
$$

and let $\gamma$ correspond to a degenerating hyperbolic element.

a) Assume $\varepsilon>0$ is sufficiently small so that $\mathcal{C}_{\ell, \varepsilon}$ is embedded in $M_{\ell}$, and, for $z \in M_{\ell}$, let $\mathcal{C}_{\ell, \varepsilon}^{z}$ denote the half of $\mathcal{C}_{\ell, \varepsilon}$ closest to $z$. Define

$$
N_{\text {hyp } ; M_{\ell}, \partial \mathcal{C}_{\ell, \varepsilon}^{z}}(T ; z)=\operatorname{card}\left\{\eta \in \Gamma_{\gamma} \backslash \Gamma \mid d_{\text {hyp }}\left(\eta z, \partial \mathcal{C}_{\ell, \varepsilon}^{z}\right)<T\right\} .
$$

Then, for any $T>0$, we have

$$
N_{\text {hyp } ; M_{\ell}, \gamma}\left(T+g\left(y_{0}, \ell\right) ; z\right)=N_{\text {hyp } ; M_{\ell}, \partial \mathcal{C}_{\ell, \varepsilon}^{z}}(T ; z) .
$$

b) For any fixed $T>0$, we have that

$$
\lim _{\ell \rightarrow 0} N_{\text {hyp } ; M_{\ell}, \gamma}\left(T+g\left(y_{0}, \ell\right) ; z\right)=N_{\text {par } ; M_{0}, P}\left(T ; z, y_{0}\right) .
$$

Proof. For fixed $\ell$, let us identify $M_{\ell}$ with a (Ford) fundamental domain in $\mathbb{H}$ such that the lift of the pinching geodesic $\gamma$ lies along the line $\operatorname{Re}(z)=0$. Then, the boundary $\partial \mathcal{C}_{\ell, \varepsilon}^{z}$ of $\mathcal{C}_{\ell, \varepsilon}$ lies along a ray $\theta(z)=$ constant. The curve $\partial \mathcal{C}_{\ell, \varepsilon}^{z}$ is orthogonal to the geodesics which transverse the sub-cylinder $\mathcal{C}_{\ell, \varepsilon}^{z}$, so $\partial \mathcal{C}_{\ell, \varepsilon}^{z}$ converges to a path on $M_{0}$ which is perpendicular to the geodesics which transverse a neighborhood of the cusp, meaning $\partial \mathcal{C}_{\ell, \varepsilon}$ converges to a horocyclic path $\mathcal{L}_{y_{0}}$ in a neighborhood of the new cusp; see Figure 2. The area of $\mathcal{C}_{\ell, \varepsilon}^{z}$ equals the area on $M_{0}$ above $\mathcal{L}_{y_{0}}$ by the choice of $\varepsilon=\varepsilon(\ell)$. By direct computation, we have

$$
\text { Area(region on } \left.M_{0} \text { above } \mathcal{L}_{y_{0}}\right)=\int_{y_{0}}^{\infty} \int_{0}^{\omega} \frac{d x d y}{y^{2}}=\frac{\omega}{y_{0}},
$$

thus we get the relation $\varepsilon=2 \omega / y_{0}$. We define $g\left(y_{0}, \ell\right)$ to be the distance from $\partial \mathcal{C}_{\ell, \varepsilon}^{z}$ 


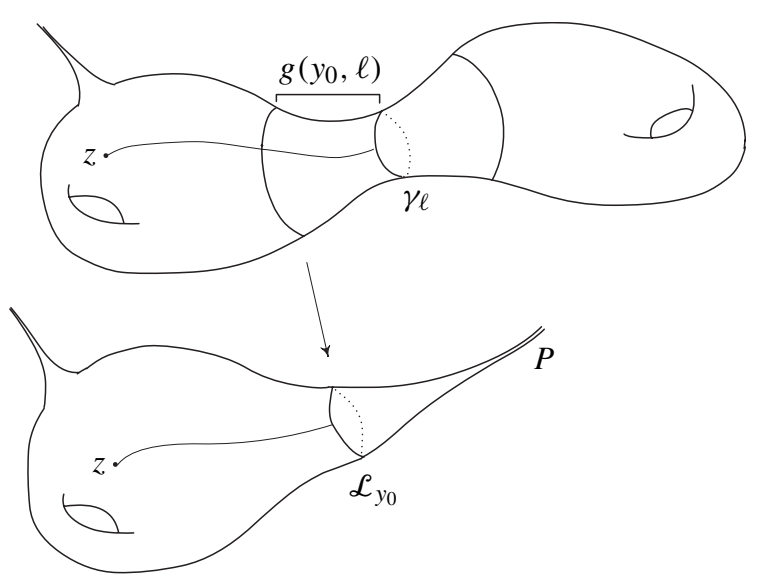

Figure 2. The Riemann surfaces $M_{\ell}$ and $M_{0}$.

to the geodesic in $\mathcal{C}_{\ell, \varepsilon}^{z}$. Using the computations from Section 2.1 and Section 2.5, we then have that

$$
g\left(y_{0}, \ell\right)=\int_{\cot ^{-1}(\varepsilon / 2 \ell)}^{\pi / 2} \frac{d \theta}{\sin \theta},
$$

which is easily evaluated, arriving at the claimed result.

Choose any $\eta \in \Gamma_{\gamma} \backslash \Gamma$. By choosing the appropriate coset representative, we may assume that $\eta z$ lies in the fundamental domain for $\Gamma_{\ell}$ from Section 2.5, meaning $0 \leq \log |\eta z|<\ell$. It is immediate that the geodesic path from $\eta z$ to the $\{\operatorname{Re}(z)=0\} \cap \mathbb{H}$ lies along the path $\rho=$ constant, which then is seen to be orthogonal to each ray $\theta=$ constant. Therefore, we have that

$$
d_{\text {hyp }}(\eta z, \gamma)=d_{\text {hyp }}\left(\eta z, \partial \mathcal{C}_{\ell, \varepsilon}^{z}\right)+d_{\text {hyp }}\left(\partial \mathcal{C}_{\ell, \varepsilon}^{z}, \gamma\right)=d_{\text {hyp }}\left(\eta z, \partial \mathcal{C}_{\ell, \varepsilon}^{z}\right)+g\left(y_{0}, \ell\right) .
$$

From the equation (12) it is clear that $d_{\mathrm{hyp}}(\eta z, \gamma)<T+g\left(y_{0}, \ell\right)$ if and only if $d_{\text {hyp }}\left(\eta z, \partial \mathcal{C}_{\ell, \varepsilon}^{z}\right)<T$, which completes the proof of part (a).

Part (b) follows from combining part (a) with the convergence of the hyperbolic metric on $M_{\ell}$ away from the developing cusps to the hyperbolic metric on $M_{0}$, as stated in Proposition 3.1.

3.4. Remark. As discussed after the proof of the main theorem, there are two cases one needs to consider in part (b) of Lemma 3.3: When the degenerating geodesic is separating, and when the degenerating geodesic is non-separating. If $\gamma$ is separating, then the statement of (b) holds without any liberty in the notation. If $\gamma$ is nonseparating, however, one needs to take into account that geodesic lengths from $z$ to $\gamma$ 
enter the cylinder about the pinching geodesic from the two different sides. The proof of (b) immediately extends to show that in the non-separating case the righthand side is actually the sum of two parabolic counting functions corresponding to the two newly formed cusps. With this noted, we choose to use the statement in (b) with its slight abuse in both the separating and non-separating cases in order to prevent burdensome notation.

3.5. Remark. As one can see, the convergence of the counting functions in Lemma 3.2 follows directly from the convergence of the hyperbolic metrics away from the developing cusps, as stated in Proposition 3.1. In Lemma 3.3, we have the added feature that the hyperbolic counting function involves the distances from the orbits of a point $z$ to the geodesic corresponding to the hyperbolic element, but the parabolic counting function involves distances to a chosen horocycle. The distances to the geodesic associated to a degenerating hyperbolic element are growing without bound; however, Lemma 3.3 can be viewed as establishing a type of "regularized convergence". To be more specific, observe that the function $g\left(y_{0}, \ell\right)$ depends solely on $y_{0}$ and $\ell$, and no other aspect of the family $M_{\ell}$. With this in mind, Lemma 3.3 states that if we "regularized" the counting functions $N_{\mathrm{hyp} ; M_{\ell}, \gamma}$ by introducing the factor $g\left(y_{0}, \ell\right)$, one then has convergence of the counting functions.

\section{Convergence of Eisenstein series}

In this section, we prove the Main Theorem. In brief, our proof uses the convergence of the counting functions for fixed $T$ (Lemma 3.2 and Lemma 3.3), the uniform bounds for the counting functions (Section 2.2) and the Stieltjes integral inequality (Section 2.6). As in Section 3, we present the arguments in the setting of a single degenerating hyperbolic element $\gamma$ whose geodesic has length $\ell$; in order to consider the general situation where there are a number of degenerating geodesics, one simply needs notational changes.

\subsection{Proof of the main theorem}

Proof of part (i). For any $T_{0}>0$, write

$$
\begin{aligned}
E_{\mathrm{hyp} ; M_{\ell}, \gamma}(s, z)= & \int_{0}^{T_{0}}(\cosh u)^{-s} d N_{\mathrm{hyp} ; M_{\ell}, \gamma}(u ; z) \\
& +\int_{T_{0}}^{\infty}(\cosh u)^{-s} d N_{\mathrm{hyp} ; M_{\ell}, \gamma}(u ; z) .
\end{aligned}
$$

Choose any $\delta>0$ and restrict $s \in \mathbb{C}$ to the half-plane $\operatorname{Re}(s) \geq 1+\delta$ for some fixed 
$\delta>0$. Trivially, we have that

$$
\left|\int_{T_{0}}^{\infty}(\cosh u)^{-s} d N_{\mathrm{hyp} ; M_{\ell}, \gamma}(u ; z)\right| \leq \int_{T_{0}}^{\infty}(\cosh u)^{-(1+\delta)} d N_{\mathrm{hyp} ; M_{\ell}, \gamma}(u ; z) .
$$

We now establish the following bound: Given any $\varepsilon>0$, there is a $T_{0}=T_{0}(\varepsilon, \delta, r)$, where $r$ is the injectivity radius at $z$, such that for each $\ell \geq 0$, we have

$$
\int_{T_{0}}^{\infty}(\cosh u)^{-(1+\delta)} d N_{\mathrm{hyp} ; M_{\ell}, \gamma}(u ; z)<\varepsilon
$$

The verification of (14) follows the proof of Lemma 1.4 from [15], which we repeat here. In the notation of Section 2.6, let $F(u)=(\cosh u)^{-(1+\delta)}$, which evidentally is real-valued, smooth, and decreasing. For $u>T_{0}$, we let

$$
\begin{aligned}
& g_{1}(u)=N_{\text {hyp } ; M_{\ell}, \gamma}(u ; z) \\
& g_{2}(u)=N_{\text {hyp } ; M_{\ell}, \gamma}\left(T_{0} ; z\right)+\frac{\sinh ^{2}\left(\frac{u+r}{2}\right)-\sinh ^{2}\left(\frac{T_{0}-r}{2}\right)}{\sinh ^{2}\left(\frac{r}{2}\right)} .
\end{aligned}
$$

As stated in Section 2.2, we have that $g_{1}(u) \leq g_{2}(u)$, and both $g_{1}$ and $g_{2}$ are realvalued and non-decreasing for $u \geq T_{0}>0$. With all this, the Stieltjes integral inequality from Section 2.6 yields the bound

$$
\begin{aligned}
& \int_{T_{0}}^{\infty}(\cosh u)^{-(1+\delta)} d N_{\mathrm{hyp} ; M_{\ell}, \gamma}(u ; z) \\
& \quad \leq \int_{T_{0}}^{\infty}(\cosh u)^{-(1+\delta)} d g_{2}(u)+\left(\cosh T_{0}\right)^{-(1+\delta)}\left\{\frac{\sinh ^{2}\left(\frac{T_{0}+r}{2}\right)-\sinh ^{2}\left(\frac{T_{0}-r}{2}\right)}{\sinh ^{2}\left(\frac{r}{2}\right)}\right\} .
\end{aligned}
$$

Elementary calculations and trigonometric identities imply that

$$
d g_{2}(u)=\frac{\sinh (u+r)}{2 \sinh ^{2}\left(\frac{r}{2}\right)} d u
$$

and

$$
\sinh ^{2}\left(\frac{T_{0}+r}{2}\right)-\sinh ^{2}\left(\frac{T_{0}-r}{2}\right)=\sinh r \sinh T_{0}
$$

Using the trivial bounds $\sinh u \leq e^{u} / 2$ and $\cosh u \geq e^{u} / 2$, we then obtain the 
estimates

$$
\begin{aligned}
& \int_{T_{0}}^{\infty}(\cosh u)^{-(1+\delta)} d g_{1}(u) \\
& \quad \leq \frac{1}{2 \sinh ^{2}\left(\frac{r}{2}\right)} \int_{T_{0}}^{\infty}(\cosh u)^{-(1+\delta)} \sinh (u+r) d u+\left(\cosh T_{0}\right)^{-(1+\delta)} \frac{\sinh r \sinh T_{0}}{\sinh ^{2}\left(\frac{r}{2}\right)} \\
& \quad \leq \frac{2^{\delta} e^{r}}{\sinh ^{2}\left(\frac{r}{2}\right)} \int_{T_{0}}^{\infty} e^{-\delta \cdot u} d u+\left(\cosh T_{0}\right)^{-(1+\delta)} \frac{\sinh r \sinh T_{0}}{\sinh ^{2}\left(\frac{r}{2}\right)} \\
& \quad \leq e^{-\delta \cdot T_{0}}\left(\frac{2^{\delta} e^{r}}{\delta \sinh ^{2}\left(\frac{r}{2}\right)}+\frac{2^{\delta} \sinh r}{\sinh ^{2}\left(\frac{r}{2}\right)}\right)
\end{aligned}
$$

which clearly can be made smaller than any $\varepsilon>0$, namely, by taking

$$
T_{0} \geq \frac{1}{\delta}\left(-\log \varepsilon+\log \left(\frac{2^{\delta} e^{r}}{\delta \sinh ^{2}\left(\frac{r}{2}\right)}+\frac{2^{\delta} \sinh r}{\sinh ^{2}\left(\frac{r}{2}\right)}\right)\right) .
$$

Therefore, we have proved the bound asserted in (14).

In addition to (16) let us assume, for convenience, that $T_{0}$ is a point of continuity of $N_{\text {hyp; } M_{0}, \gamma}(T ; z)$, meaning there is no geodesic path from $z$ to $\gamma$ on $M_{0}$ with length equal to $T_{0}$. Then, with $T_{0}$ chosen, there is an integer $N$ and an $\ell_{0}$ sufficiently small such that for $\ell<\ell_{0}$, we have $N=N_{\text {hyp } ; M_{\ell}, \gamma}\left(T_{0} ; z\right)=N_{\text {hyp } ; M_{0}, \gamma}\left(T_{0} ; z\right)$. Let $\left\{d_{k, M_{\ell}}\right\} \subset\left[0, T_{0}\right]$ be the set of lengths on $M_{\ell}$ such that for any $\eta>0$ we have

$$
N_{\text {hyp } ; M_{\ell}, \gamma}\left(d_{k, M_{\ell}}-\eta ; z\right)<N_{\text {hyp } ; M_{\ell}, \gamma}\left(d_{k, M_{\ell}}+\eta ; z\right) \text {. }
$$

For simplicity, we count the elements in the set $\left\{d_{k, M_{\ell}}\right\}$ with multiplicities so that we have

$$
\int_{0}^{T_{0}}(\cosh u)^{-s} d N_{\mathrm{hyp} ; M_{\ell}, \gamma}(u ; z)=\sum_{k=1}^{N}\left(\cosh d_{k, M_{\ell}}\right)^{-s} .
$$

With this, we can write

$$
\begin{gathered}
\int_{0}^{T_{0}}(\cosh u)^{-s} d N_{\mathrm{hyp} ; M_{\ell}, \gamma}(u ; z)-\int_{0}^{T_{0}}(\cosh u)^{-s} d N_{\mathrm{hyp} ; M_{0}, \gamma}(u ; z) \\
=\sum_{k=1}^{N}\left[\left(\cosh d_{k, M_{\ell}}\right)^{-s}-\left(\cosh d_{k, M_{0}}\right)^{-s}\right] .
\end{gathered}
$$

Observe now that the function $(\cosh u)^{-s}$ is uniformly continuous and absolutely continuous on $\left[0, T_{0}\right]$. By Lemma 3.2, which we apply for all $T<T_{0}$, there is an $\ell_{0}^{\prime}$ such that for $\ell<\ell_{0}^{\prime}$ we have

$$
\left|d_{k, M_{\ell}}-d_{k, M_{0}}\right|<\frac{\delta}{N} \quad \text { for all } k
$$


so then, $\sum_{k=1}^{N}\left|d_{k, M_{\ell}}-d_{k, M_{0}}\right|<\delta$. By the absolute continuity of $(\cosh u)^{-s}$ on $\left[0, T_{0}\right]$ we arrive at the bound

$$
\begin{aligned}
& \left|\sum_{k=1}^{N}\left[\left(\cosh d_{k, M_{\ell}}\right)^{-s}-\left(\cosh d_{k, M_{0}}\right)^{-s}\right]\right| \\
& \quad \leq \sum_{k=1}^{N}\left|\left(\cosh d_{k, M_{\ell}}\right)^{-s}-\left(\cosh d_{k, M_{0}}\right)^{-s}\right|<\varepsilon .
\end{aligned}
$$

To put all this together, let us write

$$
\begin{aligned}
& \left|E_{\mathrm{hyp} ; M_{\ell}, \gamma}(s, z)-E_{\mathrm{hyp} ; M_{0}, \gamma}(s, z)\right| \\
& \leq\left|\int_{0}^{T_{0}}(\cosh u)^{-s} d N_{\mathrm{hyp} ; M_{\ell}, \gamma}(u ; z)-\int_{0}^{T_{0}}(\cosh u)^{-s} d N_{\mathrm{hyp} ; M_{0}, \gamma}(u ; z)\right| \\
& \quad+\left|\int_{T_{0}}^{\infty}(\cosh u)^{-s} d N_{\mathrm{hyp} ; M_{\ell}, \gamma}(u ; z)\right|+\left|\int_{T_{0}}^{\infty}(\cosh u)^{-s} d N_{\mathrm{hyp} ; M_{0}, \gamma}(u ; z)\right| .
\end{aligned}
$$

The second and third terms on the right-hand side are arbitrarily small by taking $T_{0}$ as in (16), and the first term on the right-hand side is arbitrarily small by (17). With all this, the proof of part (i) of the main theorem is complete.

4.2. Remark. The referee has proposed the following alternate proof of (14). For any given $\ell$, on the geodesic $\gamma$ there are finitely many points $w_{\ell, j}, j=1, \ldots, K$ which partition $\gamma$ into segments of length $<\delta_{1}$. Since $\gamma$ is not a pinching geodesic, we can take $\gamma$, as well as the partitioning points, as lying in a subset of $M_{\ell}$ which is bounded away from the developing cusps. For any $\eta \in \Gamma_{\gamma} \backslash \Gamma$ with $d\left(\eta z, \mathscr{L}_{0}\right)<T$ on $M_{\ell}$, let $w_{\ell}$ be the point on $\gamma$ such that $d\left(\eta z, w_{\ell}\right)=d\left(\eta z, \mathscr{L}_{0}\right)$. Using that $w_{\ell}$ is within distance $\delta_{1}$ from some $w_{\ell, j}$, the triangle inequality gives the bound

$$
d\left(\eta z, w_{\ell, j}\right) \leq d\left(\eta z, w_{\ell}\right)+\delta_{1} \leq T+\delta_{1} .
$$

If we let $N_{\Gamma}(z, w, t)$ denote the counting function for the groups elements that move $z$ within distance $t$ from $w$, we then arrive at the inequality (hyperbolic lattice counting).

$$
N_{\text {hyp } ; M_{\ell}, \gamma}(T ; z) \leq \sum_{j} N_{\Gamma}\left(z, w_{\ell, j}, T+\delta_{1}\right)
$$

Using hyperbolic volume considerations, one trivially shows that $N_{\Gamma}\left(z, w_{\ell, j}, T+\delta_{1}\right)$ is bounded by $O\left(e^{T+\delta_{1}}\right)$, and the bound is uniform for the $w_{\ell, j}$ contained in a compact set. Therefore, we can write

$$
N_{\text {hyp } ; M_{\ell}, \gamma}(T ; z) \ll e^{T+\delta_{1}} .
$$


Returning to (14), one can integrate by parts to get

$$
\begin{aligned}
& \int_{T_{0}}^{\infty}(\cosh u)^{-1-\delta} d N_{\mathrm{hyp} ; M_{\ell}, \gamma}(u ; z) \\
& \quad=\left[(\cosh u)^{-1-\delta} N_{\mathrm{hyp} ; M_{\ell}, \gamma}(u ; z)\right]_{T_{0}}^{\infty} \\
& \quad+(1+\delta) \int_{T_{0}}^{\infty}(\cosh u)^{-2-\delta} \sinh u N_{\mathrm{hyp} ; M_{\ell}, \gamma}(u ; z) d u
\end{aligned}
$$

The discussion above implies that $N_{\mathrm{hyp} ; M_{\ell}, \gamma}(u ; z)$ is bounded by $O\left(e^{u+\delta_{1}}\right)$ independently of $\ell$. If we take $\delta_{1}<\delta$, then we can easily choose $T_{0}$ with the required property that the original integral is $<\varepsilon$. Indeed, the first term is $O\left(e^{\left(-\delta+\delta_{1}\right) T_{0}}\right)$ and the same applies to the second since $(\cosh u)^{-2-\delta} \sinh u=O\left(e^{(-1-\delta) u}\right)$.

As noted by the referee, an important aspect of the above argument is that one only needs the rough order of growth of $N_{\mathrm{hyp} ; M_{\ell}, \gamma}(u ; z)$, i.e. the injectivity radius plays no role in the formula.

Proof of part (ii). The proof of part (ii) follows the pattern set in the proof of part (i). The only difference is that one is considering the function $F(u)=e^{-s u}$, rather than $F(u)=(\cosh u)^{-s}$. For the integral over $\left[T_{0}, \infty\right)$, the essential feature from $F$ to be used is that $|F(u)| e^{u}$ is integrable. For the integral over $\left[0, T_{0}\right]$, one needs $F$ to be uniformly and absolutely continuous.

Proof of part (iii). We proceed as in the proof of parts (i) and (ii) with a few slight modifications. To begin, we write

$$
\begin{aligned}
E_{\mathrm{hyp} ; M_{\ell}, \gamma}(s, z)= & \int_{0}^{T_{0}+g\left(y_{0}, \ell\right)}(\cosh u)^{-s} d N_{\mathrm{hyp} ; M_{\ell}, \gamma}(u ; z) \\
& +\int_{T_{0}+g\left(y_{0}, \ell\right)}^{\infty}(\cosh u)^{-s} d N_{\mathrm{hyp} ; M_{\ell}, \gamma}(u ; z),
\end{aligned}
$$

where $g\left(y_{0}, \ell\right)$ is given in Lemma 3.3. We shall multiply both sides of (19) by $2^{-s} e^{s g\left(y_{0}, \ell\right)}$ and let $\ell$ approach zero. For the integral over $\left[T_{0}+g\left(y_{0}, \ell\right), \infty\right)$, we first use part (a) of Lemma 3.3 to write

$$
\begin{aligned}
& \int_{T_{0}+g\left(y_{0}, \ell\right)}^{\infty}(\cosh u)^{-s} d N_{\mathrm{hyp} ; M_{\ell}, \gamma}(u ; z) \\
& \quad=\int_{T_{0}}^{\infty}\left(\cosh \left(u+g\left(y_{0}, \ell\right)\right)\right)^{-s} d N_{\mathrm{hyp} ; M_{\ell}, \partial \mathfrak{C}_{\ell, \varepsilon}}(u ; z) .
\end{aligned}
$$

The geometric argument from [15] and [21] which produced (3) and (4) immediately extends to give the bound

$$
N_{\text {hyp } ; M_{\ell}, \partial \mathfrak{C}_{\ell, \varepsilon}}(u ; z) \leq N_{\text {hyp } ; M_{\ell}, \partial \mathfrak{C}_{\ell, \varepsilon}}\left(T_{0} ; z\right)+\frac{\sinh ^{2}\left(\frac{u+r}{2}\right)-\sinh ^{2}\left(\frac{T_{0}-r}{2}\right)}{\sinh ^{2}\left(\frac{r}{2}\right)},
$$


for $u>T_{0}>r$ where, as before, $r$ is the injectivity radius of $M_{\ell}$ at $z$. Following the computations in (15), we arrive at the estimate

$$
\left|2^{-s} e^{s g\left(y_{0}, \ell\right)} \int_{T_{0}+g\left(y_{0}, \ell\right)}^{\infty}(\cosh u)^{-s} d N_{\mathrm{hyp} ; M_{\ell}, \gamma}(u ; z)\right| \leq e^{-\delta \cdot T_{0}} \frac{e^{r}}{\sinh ^{2}(r / 2)}\left(\frac{1}{\delta}+1\right) .
$$

where we have written $\operatorname{Re}(s)=1+\delta$. By choosing

$$
T_{0} \geq \frac{1}{\delta}\left(-\log \varepsilon+\log \left(\frac{e^{r}}{\sinh ^{2}(r / 2)}\left(\frac{1}{\delta}+1\right)\right)\right),
$$

we have that the upper bound in (20) is less than $\varepsilon$.

For the first integral in (19), we begin by writing

$$
\begin{aligned}
\int_{0}^{T_{0}+g\left(y_{0}, \ell\right)}(\cosh u)^{-s} d N_{\mathrm{hyp} ; M_{\ell}, \gamma}(u ; z) \\
\quad=\int_{0}^{T_{0}}\left(\cosh \left(u+g\left(y_{0}, \ell\right)\right)\right)^{-s} d N_{\mathrm{hyp} ; M_{\ell}, \partial \mathcal{C}_{\ell, \varepsilon}(u ; z) .}
\end{aligned}
$$

Also, we observe the following elementary result: For fixed $x>0$ and $s \in \mathbb{C}$ with $\operatorname{Re}(s)>0$, we have

$$
\lim _{r \rightarrow \infty} 2^{-s} e^{r s}(\cosh (x+r))^{-s}=e^{-s x} .
$$

Furthermore, the limit (21) is uniform for all $x>0$ and $\operatorname{Re}(s) \geq 1+\delta$. Let $f(s, \ell)=2^{-s} y_{0}^{s} e^{s g\left(y_{0}, \ell\right)}$. Then, by Lemma 3.3 and the argument yielding (17), we have, for any $T_{0}$ as in (16), the limit

$$
\begin{array}{rl}
\lim _{\ell \rightarrow 0} & f(s, \ell) \int_{0}^{T_{0}+g\left(y_{0}, \ell\right)}(\cosh (u))^{-s} d N_{\mathrm{hyp} ; M_{\ell}, \gamma}(u ; z) \\
& =y_{0}^{s} \int_{0}^{T_{0}} e^{-s u} d N_{\mathrm{par} ; M_{0}, P}\left(u ; z, y_{0}\right) .
\end{array}
$$

We now use (20) and (22) and the triangle inequality, as in (18), in order to prove

$$
\lim _{\ell \rightarrow 0} f(s, \ell) E_{\mathrm{hyp} ; M_{\ell}, \gamma}(s, z)=\omega^{s} E_{\mathrm{par} ; M_{0}, P}(s, z) .
$$

To complete the proof of part (iii), it remains to evaluate $f(s, \ell)$.

Evaluation of $f(s, \ell)$. As shown in the proof of Lemma 3.3, we have

$$
g\left(y_{0}, \ell\right)=\log \left(\frac{\omega}{y_{0} \ell}+\sqrt{\left(\frac{\omega}{y_{0} \ell}\right)^{2}+1}\right),
$$


from which we immediately derive the relation

$$
f(s, \ell)=2^{-s} y_{0}^{s}\left(\frac{\omega}{y_{0} \ell}+\sqrt{\left(\frac{\omega}{y_{0} \ell}\right)^{2}+1}\right)^{s}=(\omega / \ell)^{s}+o\left((\omega / \ell)^{s}\right) \quad \text { as } \ell \rightarrow 0 .
$$

Substituting (24) into (23), then multiplying both sides by $\omega^{-s}$, completes the proof of part (iii) of our Main Theorem.

4.3. Remark. In the setting of part (iii) of our Main Theorem, consider the differential equation satisfied by $E_{\mathrm{hyp} ; M_{\ell}, \gamma}(s, z)$ which, after multiplying by $\ell^{-s}$, is the identity

$$
\begin{aligned}
& \Delta\left(\ell^{-s} E_{\mathrm{hyp} ; M, \gamma}(s, z)\right) \\
& \quad=s(1-s)\left(\ell^{-s} E_{\mathrm{hyp} ; M, \gamma}(s, z)\right)+(s \ell)^{2}\left(\ell^{-s-2} E_{\mathrm{hyp} ; M, \gamma}(s+2, z)\right) .
\end{aligned}
$$

By part (iii) of our Main Theorem, we have that

$$
\begin{aligned}
\lim _{\ell \rightarrow 0} & \left(s(1-s)\left(\ell^{-s} E_{\mathrm{hyp} ; M, \gamma}(s, z)\right)+s^{2}\left(\ell^{-s-2} E_{\mathrm{hyp} ; M, \gamma}(s+2, z)\right) \cdot \ell^{2}\right) \\
& =s(1-s) E_{\mathrm{par} ; M_{0}, P}(s, z),
\end{aligned}
$$

for all $\operatorname{Re}(s)>1$ and $z$ bounded away from the developing cusps. The point here is that the second term on the right-hand side of (25) vanishes through degeneration. Heuristically, this shows that in the setting of part (iii), the differential equation for the hyperbolic Eisenstein series limits to the differential equation for the parabolic Eisenstein series.

4.4. Remark. In the definition of the parabolic Eisenstein series (5) we included a multiplicative factor of $\omega^{-s}$. Analogously, we could have included a factor of $\ell_{\gamma}^{-s}$ in the definition of the hyperbolic Eisenstein series (7). Let us use the term adjusted hyperbolic Eisenstein series to denote the hyperbolic Eisenstein series from (7) multiplied by $\ell_{\gamma}^{-s}$. With this factor, then part (iii) of the Main Theorem states that the adjusted hyperbolic Eisenstein series associated to the degenerating hyperbolic element converges to the parabolic Eisenstein series of the newly formed cusp. In addition, the adjusted hyperbolic Eisenstein series will satisfy an equation similar to (9), where the second term has the multiplicative factor of $\left(s \ell_{\gamma}\right)^{2}$, as in (25).

4.5. Remark. The concept of an Eisenstein series associated to an elliptic element of $\Gamma$ was first defined in [12] and has been studied in [22]. At this time, A. von Pippich is continuing her systematic investigation of elliptic Eisenstein series, which, almost certainly, will include convergence results as in the present paper when considering a sequence of elliptically degenerating Riemann surfaces. This problem in under investigation by the first named author of this paper (D.G.) and von Pippich. 
4.6. Remark. After completion and initial review of this article, Gautam Chinta called our attention to the article [3] where the author establishes the Main Theorem using different techniques. The advantage of our approach is the introduction of counting function techniques when studying Eisenstein series, both parabolic and hyperbolic, thus reducing the main theorem to convergence questions associated to the various counting functions.

Acknowledgements. The authors thank Anupam Bhatnagar, James Cogdell, Jozek Dodziuk, and James Harlacher for numerous helpful conversations. In particular, we thank Cogdell for several comments concerning exposition as well as the contents of Remark 4.4. We also thank the referee for providing an extensive list of comments which enhanced the presentation and clarity of the paper. The second named author (J.J.) acknowledges support from NSF grants and several PSC-CUNY awards.

\section{References}

[1] W. Abikoff, Degenerating families of Riemann surfaces. Ann. of Math. 105 (1977), 29-44. Zbl 0347.32010 MR 0442293

[2] J. Fay, Theta functions on Riemann surfaces. Lecture Notes in Math. 352, Springer-Verlag, Berlin 1973. Zbl 0281.30013 MR 0335789

[3] T. Falliero, Dégénérescence de séries d'Eisenstein hyperboliques. Math. Ann. 339 (2007), 341-375. Zbl 05208130 MR 2324723

[4] D. Hejhal, The Selberg trace formula for PSL(2, R). Vol. 2, Lecture Notes in Math. 1001, Springer-Verlag, Berlin 1983. Zbl 0543.10020 MR 0711197

[5] D. Hejhal, Regular $b$-groups, degenerating Riemann surfaces, and spectral theory. Mem. Amer. Math. Soc. 437, Amer. Math. Soc., Providence, RI, 1990. Zbl 0718.11024 MR 1052555

[6] J. Huntley, J. Jorgenson and R. Lundelius, On the asymptotic behavior of counting functions associated to degenerating hyperbolic Riemann surfaces. J. Funct. Analysis 149 (1997), 58-82. Zbl 0889.58078 MR 1471099

[7] H. Iwaniec, Spectral methods of automorphic forms. Second edition. Grad. Stud. in Math. 53, Amer. Math. Soc., Providence, RI; Revista Matemática Iberoamericana, Madrid 2002. Zbl 1006.11024 MR 1942691

[8] L. Ji and M. Zworski, The remainder estimate in spectral accumulation for degenerating hyperbolic surfaces. J. Funct. Anal. 114 (1993), 412-420. Zbl 0783.58078 MR 1223708

[9] J. Jorgenson, Asymptotic behavior of Faltings's delta function. Duke Math. J. 61 (1990), 221-254. Zbl 0746.30032 MR 1068387

[10] J. Jorgenson and J. Kramer, Bounds for special values of Selberg zeta functions of Riemann surfaces. J. Reine Angew. Math. 541 (2001), 1-28. Zbl 0986.11058 MR 1876283

[11] J. Jorgenson and J. Kramer, Non-completeness of the Arakelov-induced metric on moduli space of curves. Manuscripta Math. 119 (2006), Zbl 1096.14017 MR 2223627

[12] J. Jorgenson and J. Kramer, Canonical metrics, hyperbolic metrics, and Eisenstein series for $\mathrm{PSL}_{2}(\mathbf{R})$, in preparation (unfinished manuscript, (2003)). 
Vol. 83 (2008) On the appearance of Eisenstein series through degeneration

[13] J. Jorgenson and R. Lundelius, Convergence of the heat kernel and the resolvent kernel on degenerating hyperbolic Riemann surfaces of finite volume. Quaestiones Math. 18 (1995), 345-363. Zbl 0853.58099 MR 1354117

[14] J. Jorgenson and R. Lundelius, Convergence theorems for relative spectral functions on hyperbolic Riemann surfaces of finite volume. Duke Math. J. 80 (1995), 785-819. Zbl 0973.58016 MR 1370116

[15] J. Jorgenson and R. Lundelius, Convergence of the normalized spectral counting function on degenerating hyperbolic Riemann surfaces of finite volume. J. Funct. Anal. 149 (1997), 25-57. Zbl 0887.58057 MR 1471098

[16] J. Jorgenson and R. Lundelius, regularized heat trace for hyperbolic Riemann surfaces of finite volume. Comment. Math. Helv. 72 (1997), 636-659. Zbl 0902.58040 MR 1600164

[17] C. Judge, Tracking eigenvalues to the frontier of moduli space I: Convergence and spectral accumulation. J. Funct. Anal. 184 (2001), 273-290. Zbl 1005.58012 MR 1850999

[18] C. Judge, Tracking eigenvalues to the frontier of moduli space II: Limits for eigenvalue branches. Geom. Funct. Anal. 12 (2002), 93-120. Zbl 1023.58017 MR 1904559

[19] T. Kubota, Elementary theory of Eisenstein series. Kodansha Ltd., Tokyo; Halsted Press [John Wiley \& Sons], New York, London, Sydney 1973. Zbl 0268.10012 MR 0429749

[20] S. Kudla and J. Millson, Harmonic differentials and closed geodesics on a Riemann surface. Invent. Math. 54 (1979), 193-211. Zbl 0429.30038 MR 0553218

[21] R. Lundelius, Asymptotics of the determinant of the Laplacian on hyperbolic surfaces of finite volume. Duke Math. J. 71 (1993), 211-242. Zbl 0790.58044 MR 1230291

[22] A.-M. v. Pippich, Elliptische Eisensteinreihen. Diplomarbeit, Humboldt-Universität zu Berlin, 2005.

[23] B. Randol, Cylinders in Riemann surfaces. Comment. Math. Helv. 54 (1979), 1-5. Zbl 0401.30036 MR 0522028

[24] M. Risager, On the distribution of modular symbols for compact surfaces. Internat. Math. Res. Notices 41 (2004), 2125-2146. Zbl 02150556 MR 2078851

[25] S., Wolpert, Disappearance of cusp forms in special families. Ann. of Math. 139 (1994), 239-291. Zbl 0826.11024 MR 1274093

Received July 19, 2006

Daniel Garbin, Mathematics Ph.D. Program, The Graduate Center of CUNY, 365 Fifth Avenue, New York, NY, U.S.A.

E-mail: daniel_garbin@yahoo.com

Jay Jorgenson, Department of Mathematics, The City College of New York, Convent Avenue at 138th Street, New York, NY 10031, U.S.A.

E-mail: jjorgenson@mindspring.com

Michael Munn, Mathematics Ph.D. Program, The Graduate Center of CUNY, 365 Fifth Avenue, New York, NY, U.S.A.

E-mail: mikemunn@gmail.com 